\section{Induced suction and lithospheric plate pall}

SHOEMAKER ${ }^{1}$ proposes to explain the driving force of plate tectonics as 'induced suction' by the low density sediments in the trench. However, his calculation, which is based solely on fluid statics, does not tell us what the energy source for the motion is, neither does it tell us anything essentially new about subduction dynamics.

To illustrate this, consider an experiment in which a high viscosity fluid is flowing steadily over the edge of a horizontal table. The fluid which has just passed over the edge responds to the unbalanced vertical force of gravity, and this force is 'communicated' to the rest of the fluid by means of a horizontal pressure gradient (that is, the upper surface of the fluid is no longer horizontal). A statics calculation of the type made by Shoemaker would demonstrate that the table edge provides 'suction', but this obscures the fact that the cause of the motion is the vertical force of gravity. The essentials of the argument still apply if the rheology is more complicated.

In the plate tectonics context, the value Shoemaker obtains for the 'suction' is nothing more than the downward force on the subducting slab, presumably caused by gravity $^{2}$. Trench depression can be predicted by a dynamical calculation ${ }^{3}$, and the accumulation of sediments at the trench is an effect of plate motion, not a cause.

\section{J. Stevenson}

Research School of Earth Sciences, Australian National University, Canberra, Australia 2600

1. Shoemaker, E. M. Nature 272, 241 (1978).

2. Stevenson, D. J. \& Turner, J. S. Nature 270, 334 (1977) 3. McKenzie, D. P. Geophys. J.R. astr. Soc. 18, 18 (1969)

SHOEMAKER REPLIES-Induced suction ${ }^{1}$ is a consequence of the combination of two conditions being satisfied by the subduction process: first, that the fluids or materials above and below the subducting plate have different densities and second, that the subducting plate bends beneath the overriding plate in contrast to the idealised situation where it passes from the horizontal to the downward dipping state through a sharp corner ${ }^{2}$. The force of induced suction is not the downward force of the subducting slab nor is there any direct connection between these two forces. There is an obvious indirect connection in that the downward force of the subducting slab has a major influence in creating the downward bend which causes the induced suction.

The major energy source for plate motion may be downward pull of the subducting slab; it might be something else. However, any consideration of energy is immaterial to the argument presented for 'deriving' the existence of induced suction. The argument was based on equilibrium which has equal validity with energy principles.

There is no connection between the example of a highly viscous fluid flowing over the edge of a table and induced suction. The 'communication' between the downward flowing fluid and the horizontal flowing fluid on the table is provided by the table edge; the horizontal component of the force exerted by the table edge upon the fluid pulls the fluid along the table. This is not induced suction. Moreover, the fluid will certainly flow if, for example, there is air above and below the fluid 'slab'.

\section{E. M. SHOEMAKER}

Faculty of Science,

Simon Fraser University

Burnaby, British Columbia V5A 1 S6

1. Shoemaker, E. M. Nature 272, 241 (1978)

2. Stevenson, D. J. \& Turner, J. S. Nature 270, 334 (1977).

\section{Circadian variation in immune response to oxazolone}

FACHET AND ANDO $^{1}$ suggest that cellular and humoral responses to oxazolone are under the control of $\mathrm{H}-2$ genes. Their evidence for the control of cellular immunity was based on the differences between inbred and $\mathrm{H}-2$ congeneic strains of mice in the magnitude of the delayed hypersensitivity response to oxazolone. This was measured in the ears of sensitised mice as the difference in ear thickness measured immediately before and $24 \mathrm{~h}$ after challenge. Using this method, we have demonstrated in rats that the magnitude of the maximum immune response depends on the time of day at which oxazolone is applied to the ears ${ }^{2}$. Subsequently, we have shown that there are circadian variations in the skin response to the injection of purified protein derivative in $\operatorname{man}^{3}$. It seems that the time of day at which the antigen is encountered influences the magnitude of the delayed hypersensitivity response.

Most investigators carry out experiments at the same time of the day. We would welcome an assurance from Fachet and Ando on this point, for if their experiments were not standardised to one time of challenge for all the mouse strains, this affects the interpretation of their findings. Additionally, if the oxazolone challenges were given over a long time span wwithin a single day this might affect the results adversely, as the maximum observed response to challenge with oxazolone at 10.00 in the rat is over eight times the minimum response at 16.00 .
Data on the time of challenge and the lighting regimen of the animals are not available in the paper from the Hungarian Unit which therefore does not contain sufficient details for the experiments to be repeated, surely a prerequisite of any scientific publication.

Biological rhythms influence many cellmediated and humoral immune processes with effects which are often of a high order of magnitude. Our recent finding (unpublished) that the delayed hypersensitivity response to oxazolone can be manipulated by altering the lighting regimen of the animals is a further demonstration that the basic principles of chronobiological experimentation need to be introduced immediately into immunological studies.

\section{R. POWNALL}

\section{J. R. COVE-SMITH}

TRICIA A. KABLER

INGE KOWANKO

\section{S. KNAPP}

Chronotherapeutic Research Group, Renal Unit, City Hospital, Hucknall Road, Nottingham, UK

1. Fachet, J. \& Ando, I. Nature 273, 239-240 (1978)

2. Pownall, R. \& Knapp, M. S. Clin. Sci. molec. Med. 54, 447-449 (1978).

3. Cove-Smith, J. R., Pownall, R., Kabler, P. A. \& Knapp, M. S. Br. med. J. 2, 253-254 (1978).

FACHET AND ANDÓ REPLY-The points raised by Pownall et al. are of interest and we make the following comments. All experimental mice of the $\mathrm{H}-2$ congeneic strains used were sensitised and their delayed-type hypersensitivity (DTH) to oxazolone was tested between 09.00 and 11.00 . The same timing of experimental procedures was applied when the DTH to oxazolone was tested in a larger number of animals of the same strain and in mice of other inbred mouse strains. The same conclusion was reached concerning the effect of genes within and outside the $H-2$ complex. A single strain has not been independently tested; a series of different strains only has been investigated and used for comparison ${ }^{1}$.

Within the scope of our experiments we found no diurnal variation in responsiveness on testing $\mathrm{H}-2$ congeneic mouse strains with oxazolone but it well might occur in different conditions.

\section{J. FACHET}

\section{ANDó}

\section{Institute of Genetics,}

Biological Research Center,

Hungarian Academy of Sciences,

PO Box 521, H-6701 Szeged, Hungary

1. Fachet, J. \& Andó, I. Eur. J. Immun. 7, 223-226, 516-519 (1977); 8, 101-104 (1978). 